\title{
Clinical associations with immature breathing in preterm infants: part 2-periodic breathing
}

\author{
Manisha Patel', Mary Mohr², Douglas Lake ${ }^{3}$, John Delos², J. Randall Moorman³, Robert A. Sinkin', John Kattwinkel' and \\ Karen Fairchild ${ }^{1}$
}

BACKGROUND: Periodic breathing (PB) is a normal immature breathing pattern in neonates that, if extreme, may be associated with pathologic conditions.

METHODS: We used our automated PB detection system to analyze all bedside monitor chest impedance data on all infants $<35$ wk' gestation in the University of Virginia Neonatal Intensive Care Unit from 2009-2014 ( $n=1,211)$. Percent time spent in PB was calculated hourly ( $>50$ infant-years' data). Extreme PB was identified as a $12-\mathrm{h}$ period with $\mathrm{PB}>6$ SDs above the mean for gestational age (GA) and postmenstrual age and $>10 \%$ time in PB.

RESULTS: PB increased with $G A$, with the highest amount in infants 30-33 wk' GA at about 2 wk' chronologic age. Extreme PB was identified in 76 infants and in $45 \%$ was temporally associated with clinical events including infection or necrotizing enterocolitis (NEC), immunizations, or caffeine discontinuation. In 8 out of 28 cases of septicemia and 10 out of 21 cases of NEC, there was a $>2$-fold increase in \%PB over baseline on the day prior to diagnosis.

CONCLUSION: Infants <35 wk GA spend, on average, $<6 \%$ of the time in PB. An acute increase in PB may reflect illness or physiological stressors or may occur without any apparent clinical event.

$\mathbf{P}$ eriodic breathing $(\mathrm{PB})$, repetitive short cycles of respiratory pauses and breathing, is a normal physiologic breathing pattern that occurs during sleep in most newborns $(1,2)$. Although publications characterizing PB in infants in the neonatal intensive care unit (NICU) began to appear more than a half century ago $(3,4)$, our understanding of the epidemiology and associations with this breathing pattern remain incomplete, in part due to the complexity of analyzing breathing in sufficiently large numbers of infants over long periods of time.

$\mathrm{PB}$ is known to be distinct from apnea of prematurity (AOP) in that it occurs in term as well as preterm infants, peaks later, and lasts longer $(2,5)$. It typically appears in the second week after birth, peaks at several weeks of age, then decreases but may continue for up to 6 mo or longer $(2,6)$. In contrast, AOP begins within a day or two after birth, gradually diminishes as preterm infants mature, and usually resolves around 36-40 wk' postmenstrual age (PMA) (7). Both immature breathing patterns are thought to reflect altered sensitivity of chemoreceptors to changes in blood oxygen and carbon dioxide content (8-13), with increased "gain" in the receptors identified as a major contributing factor to the oscillations between short apneic pauses and breathing characteristic of $\mathrm{PB}$. AOP is often associated with a significant decline in heart rate (HR) and oxygen saturation $\left(\mathrm{SpO}_{2}\right)$ leading to "ABD" events (apnea, bradycardia, desaturation) experienced by nearly all very preterm infants while in the NICU. In PB the short apneic pauses may be associated with small declines in $\mathrm{HR}$ or $\mathrm{SpO}_{2}$ but usually not to a level that would trigger bedside monitor alarms (1416). Although $P B$ has historically been considered to be benign and not requiring treatment, a recent study reported a decline in cerebral oxygenation in a small number of preterm infants during the apneic phase of $\mathrm{PB}$, highlighting the need for further studies into the course and consequences of $\mathrm{PB}$ (17).

Physiologic PB is not seen in healthy children or adults except with exposure to acute hypoxia on ascending to high altitude (18). Pathologic forms of PB occur in adults with acute or chronic illness, including Cheyne-Stokes respiration in congestive heart failure (19), and central PB following brain injury such as stroke (20). These forms of $\mathrm{PB}$ are linked to poor outcomes and mortality $(21,22)$. The mechanisms of pathology associated PB in adults are not known with certainty, but mathematical modeling and some physiologic studies in animals and humans have implicated increased loop gain (abnormal chemoreceptor sensitivity or prolonged circulatory time from lung to chemoreceptors) (23-25). In neonates, pathologic PB has not been well described. We observed a number of cases in which infants spent an extremely large proportion of time in PB either acutely (hours) or chronically (weeks) and subsequently experienced adverse events (26), and we therefore sought to study PB in a comprehensive way.

Prior studies of PB have been limited by constraints in ability to store and analyze large amounts of cardiorespiratory data, but recent advances in data acquisition, storage, and processing 
allowed our research group to develop a method to measure PB that underwent extensive validation and was found to have $96 \%$ sensitivity and $90 \%$ specificity for detecting PB identified by neonatologists reviewing chest impedance and vital sign data (26). The goals of the current study were to use our automated system for detecting $\mathrm{PB}$ to quantify physiologic $\mathrm{PB}$ in a large cohort of preterm infants $<35$ wk' gestation throughout their entire NICU course and to identify clinical correlates of extreme PB in order to begin to understand pathology-associated $\mathrm{PB}$ in neonates.

\section{RESULTS}

\section{Demographics and Quantitation of PB}

In the 5-year study period, 1,372 infants $<35$ wk' gestation were admitted to the University of Virginia NICU, and some chest impedance waveform data were available for analysis in 1,268 infants. Of these, 57 were excluded because they had no chest impedance data off mechanical ventilation and prior to term PMA, either due to death or transfer from our unit. Demographics and type of respiratory support throughout the NICU stay are shown in the companion paper on apnea (Fairchild et al., (27)). Gestational age distribution of the 1,211 infants with PB analyzed was 190 infants <27 wk GA, 297 from 27-30 wk GA, and 724 infants from 31-34wk GA.

Median number of days analyzed per infant was 15 (25th75th percentile 7-34 d) which corresponds to approximately 53 infant-years of data. Figure 1 is a graphic representation of $\mathrm{PB}$, with repetitive respiratory pause/breath cycles in both the unfiltered and filtered (motion and cardiac artifact removed) chest impedance waveforms. The respiratory pauses in PB sometimes lead to declines in heart rate and/or oxygen saturation, as shown in Figure $\mathbf{1 b}$.

Figure 2 shows a heat map representation of mean daily $\% \mathrm{~PB}$ for each week of GA and PMA, with warmer colors indicating higher $\mathrm{PB}$ peaking at about 5\% around 2-4 wk' chronologic age for infants of higher GA. Percentage of time spent in PB generally increases with gestational age, with infants $<27 \mathrm{wk}$ GA spending, on average, only about $1 \%$ of the time in $\mathrm{PB}$.

\section{Clinical Associations With Extreme PB}

Extreme $\mathrm{PB}$, defined as at least one 12-h episode with $z$-score $>6$ and $>10 \%$ time spent in PB, was identified in 76 infants. Table 1 compares the clinical characteristics of infants with and without extreme PB. Medical records were reviewed to identify clinical events corresponding to the $12 \mathrm{~h}$ extreme $\mathrm{PB}$ episode with the highest $z$-score for each of these 76 infants. Suspected or proven infection or necrotizing enterocolitis (NEC) occurred within 24 hours of the extreme PB episode in 11 cases (14\%), immunizations or surgery within 24 hours prior in 12 cases (16\%), and discontinuation of caffeine within $8 \mathrm{~d}$ prior in 11 cases (14\%) (Figure 3). We also randomly selected 76 GA-matched infants with PMA-matched time periods not demonstrating extreme $\mathrm{PB}$. On medical record review, we identified suspected or proven infection or NEC within $24 \mathrm{~h}$ in 2 cases (2.6\%), surgery or immunizations within $24 \mathrm{~h}$ in 5 cases $(6.6 \%)$, and discontinuation of caffeine within $8 \mathrm{~d}$ prior in 6 cases (7.9\%). Thus, clinical conditions were significantly more likely to be identified around times of extreme PB $(34 / 76$ or $45 \%)$ compared to times of no extreme $\mathrm{PB}(13 / 76$ or $17 \% ; P<0.001)$.

Of note, one infant with extreme $\mathrm{PB}$ died of sudden infant death syndrome (SIDS) $5 \mathrm{wk}$ after NICU discharge. We reviewed matched birth/death certificates for the state of Virginia during the date range of the study, and this was the only preterm infant discharged from the UVA NICU known to have died of SIDS. We previously reported that this infant spent over fivefold more time in PB throughout her NICU stay compared to the average for all other $32 \mathrm{wk}$ GA infants (26). a

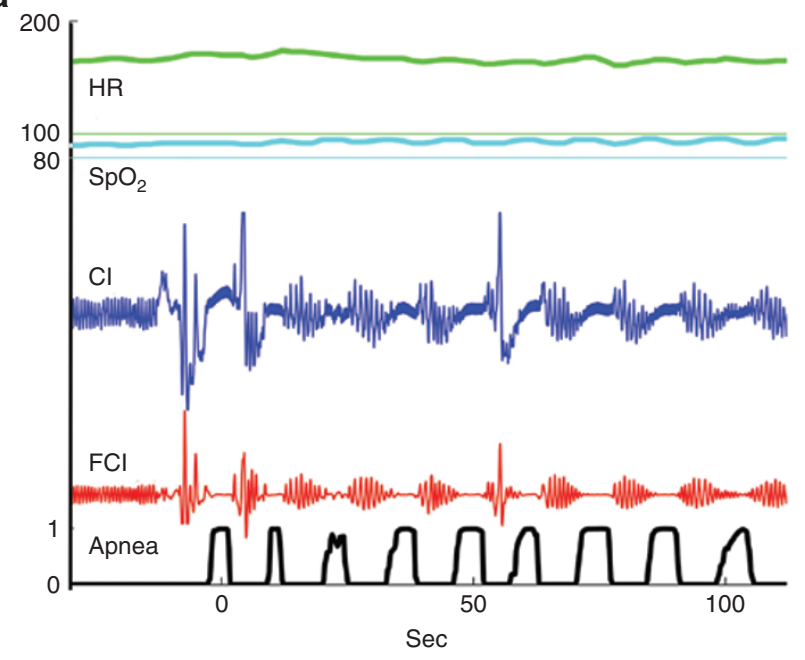

b

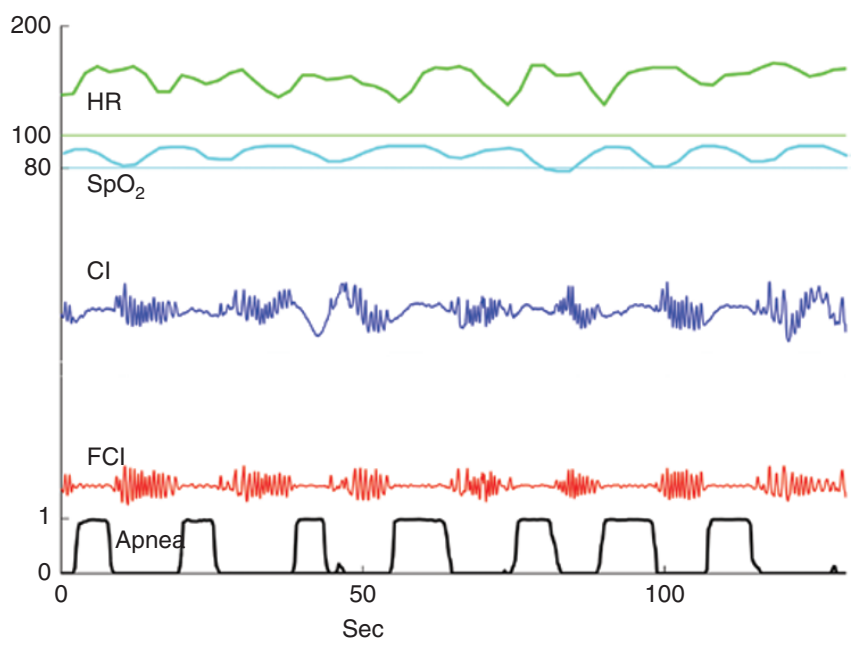

Figure 1 Periodic breathing examples. Chest impedance waveform data from NICU bedside monitors were analyzed for PB. Graphic representations are shown of $2 \mathrm{~min}$ of vital sign and waveform data. From top to bottom: heart rate (HR with 100 beats/min indicated by the thin green line), oxygen saturation $\left(\mathrm{SpO}_{2}\right.$ with $80 \%$ indicated by the thin blue line), chest impedance $(\mathrm{Cl})$, filtered chest impedance ( $\left.\mathrm{FCl}\right)$, and computer algorithm-detected probability of apnea based on low variance in the filtered $\mathrm{Cl}$ signal as previously reported (27). A wavelet transform analysis of short apneic pauses was previously validated for automated analysis of \%time spent in PB (26). (a) Example of periodic breathing without significant changes in heart rate or $\mathrm{SpO}_{2}$. (b) Example of periodic breathing with decreases in heart rate and $\mathrm{SpO}_{2}$ corresponding to the respiratory pauses. 


\section{Articles $\mid$ Pateletal.}

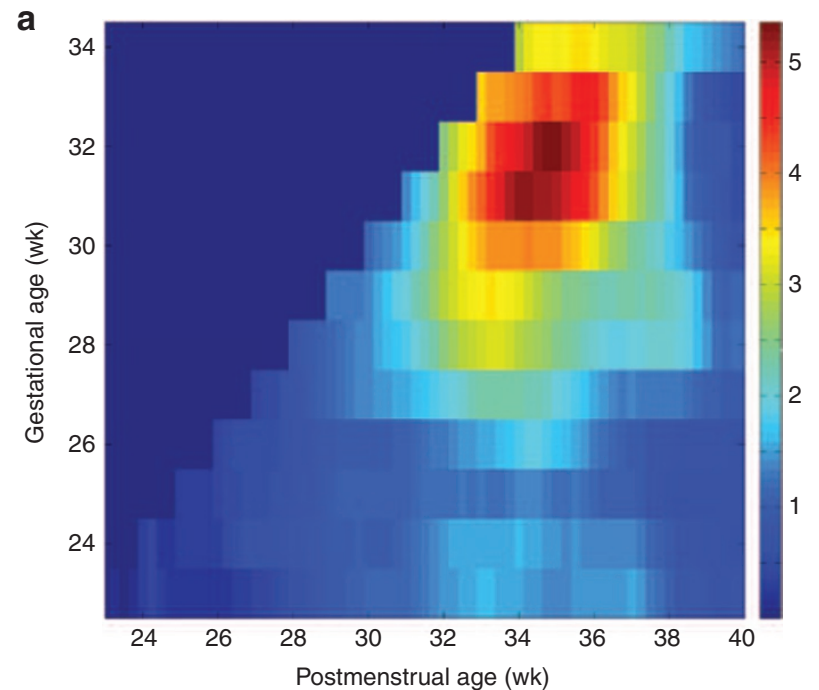

b

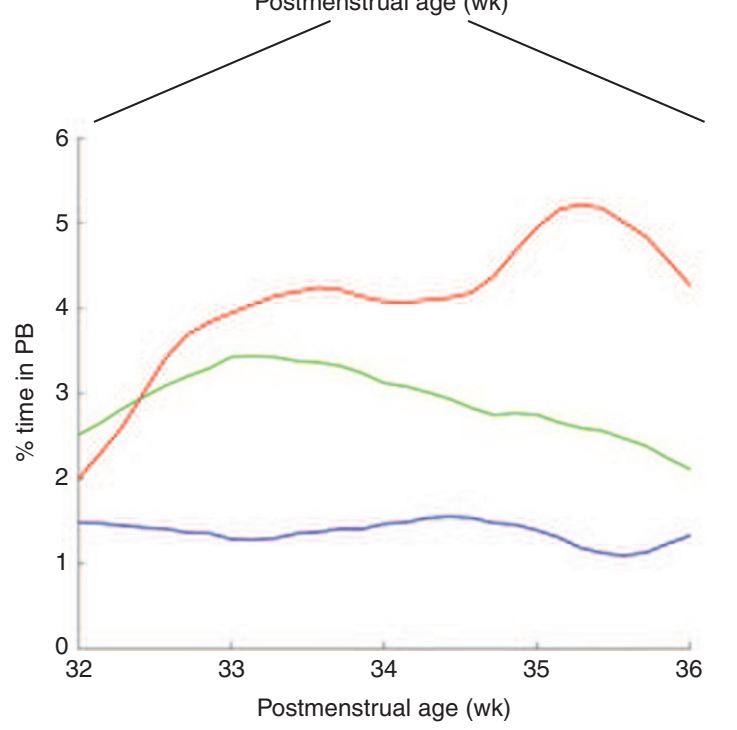

Figure 2 Percentage of time spent in PB per day of data, based on gestational and postmenstrual age. PB was analyzed during all times that chest impedance data were available and infants were not on mechanical ventilation for all infants $<35 w^{\prime} G A(n=1,211)$. (a) Heat map showing mean daily \% time in PB, based on GA and PMA. The color scale goes from blue (mean 0\% PB per day) to red (mean 5\% PB per day). (b) Percent PB from 32 to 36 wk PMA in three GA groups, <27 wk (blue), 27-30 wk (green), and $31-34 \mathrm{wk}$ (red).

\section{Increased PB in Infants With NEC and Septicemia}

Since the extreme PB analysis identified suspected or proven infection-related illnesses as being temporally associated with a high percentage of time spent in $\mathrm{PB}$, we identified all cases of late-onset septicemia (LOS) and NEC to determine whether infants demonstrated an increase in $\mathrm{PB}$ in the day prior to diagnosis over their prior day's baseline. There were 28 cases of LOS and 21 cases of NEC in infants not on a ventilator and with chest impedance data available for PB analysis at least $50 \%$ of the time in the $2 \mathrm{~d}$ leading up to diagnosis. Figure 4 shows \% PB $2 \mathrm{~d}$ compared to $1 \mathrm{~d}$ prior to diagnosis. $\mathrm{A}>2$-fold increase in $\mathrm{PB}$ occurred in the 24 -h period prior to diagnosis in 10 out of 21 cases of NEC (48\%) and 8 out of 28 cases of $\operatorname{LOS}(29 \%)$.
Table 1 Characteristics of infants with and without extreme periodic breathing $^{\text {a }}$

\begin{tabular}{lccc}
\hline $\begin{array}{l}\text { Demographics, } \\
\text { morbidities }\end{array}$ & $\begin{array}{c}\text { No extreme PB } \\
(n=1,135)\end{array}$ & $\begin{array}{c}>12 \text {-h extreme } \\
\mathrm{PB}^{\mathrm{a}}(n=76)\end{array}$ & $P^{*}$ \\
\hline $\begin{array}{l}\text { Gestational age, } \\
\text { weeks }\end{array}$ & $32(29-33)$ & $28(25-30)$ & $<0.001$ \\
$\begin{array}{l}\text { Birthweight, grams } \\
\text { IVH grade III-IV }\end{array}$ & $\begin{array}{c}1,640(1,140-2,070) \\
\text { Bronchopulmonary }\end{array}$ & $\begin{array}{c}1,075(833-1,440) \\
\text { dysplasia }\end{array}$ & 60.001 \\
$\begin{array}{l}\text { Late-onset } \\
\text { septicemia }\end{array}$ & $79(7.9 \%)$ & $26(34 \%)$ & $\mathrm{ns}^{*}$ \\
$\begin{array}{l}\text { Necrotizing } \\
\text { enterocolitis }\end{array}$ & $31(2.7 \%)$ & $10(11.8 \%)$ & $\mathrm{ns}^{*}$ \\
$\begin{array}{l}\text { Death prior to NICU } \\
\text { discharge }\end{array}$ & $17(1.5 \%)$ & $9(11.8 \%)$ & $<0.05^{*}$ \\
\hline
\end{tabular}

Data are presented as median (25th-75th percentile) or $n(\%)$.

GA, gestational age; IVH, intraventricular hemorrhage; ns, not significant; PB, periodic breathing.

at least 12 hours of PB $z$-score $>6$ and $>10 \%$ time in PB. *Adjusted for GA where indicated.

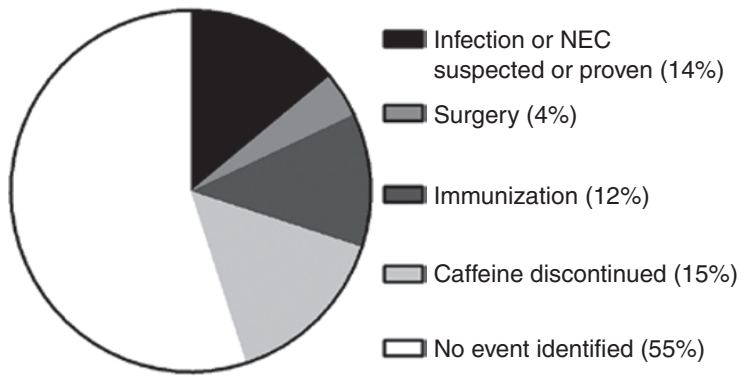

Figure 3 Clinical events near the time of extreme PB. Seventy-six infants had extreme $\mathrm{PB}$, defined as at least one 12-h period during which the amount of time in PB was $>6$ SDs above the mean for that GA and PMA ( $z$-score $>6$ ), and $>10 \%$ of the time in PB. For the highest PB period for each infant, chart review was performed to identify temporally associated clinical events. Events were identified in $45 \%$ of cases, including suspected or proven sepsis or NEC within $\pm 24 \mathrm{~h}$, surgery or immunizations within $24 \mathrm{~h}$ prior, or caffeine discontinued within $8 \mathrm{~d}$ prior.

\section{Incidence and Timing of PB Relative to AOP}

We used our automated apnea detection system (28) to quantify central AOP in the same 1,211 infants on whom PB analysis was performed. "ABD" events, central apnea events lasting at least $10 \mathrm{~s}$ and with associated bradycardia ( $<100$ beats/ $\min )$ and oxygen desaturation $\left(\mathrm{SpO}_{2}<80 \%\right)$, were measured. Figure 5 shows the relative incidence and timing of $\mathrm{PB}$ compared with AOP, from 26 to 36 wk PMA. PB occurs in nearly all preterm infants at all GA, whereas AOP is less common among higher GA preterm infants. PB tends to appear a week after birth, peaks at several weeks of age, and then gradually declines but persists beyond NICU discharge. In contrast, AOP begins shortly after birth and declines steadily after $30 \mathrm{wk}$ PMA. Although significant events requiring intervention typically resolve near term corrected age, we found that short ABD10 events persist in some infants near the time of NICU discharge (see companion paper, Fairchild et al., (27)). 


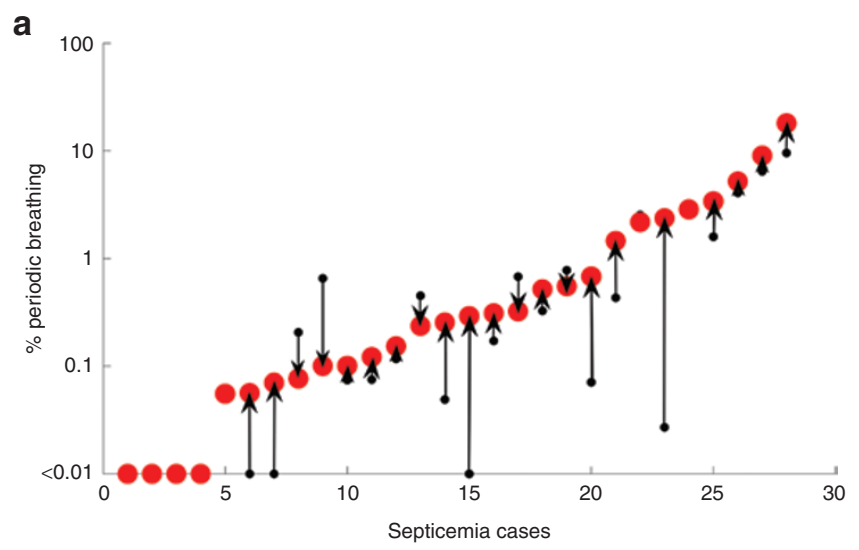

b

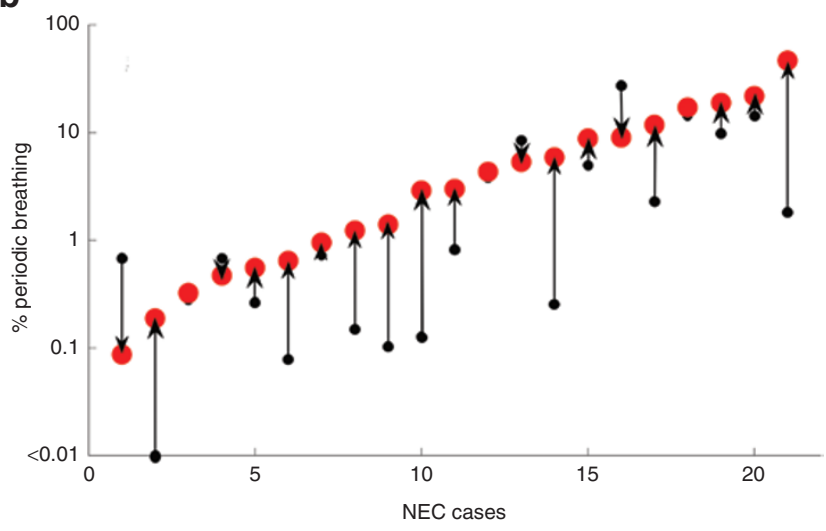

Figure 4 PB prior to episodes of septicemia and NEC. In each case of (a) late-onset septicemia or (b) necrotizing enterocolitis in which infants were not on mechanical ventilation and chest impedance data were available, $\%$ time in PB is shown in the 24-48-h period prior to diagnosis (small black dot), and the 0-24-h period prior to diagnosis (large red dot). Arrows indicate the magnitude and direction of change. In $29 \%$ of septicemia cases and $48 \%$ of NEC cases, there was a $>2$-fold increase in $\% \mathrm{~PB}$ in the day prior to diagnosis compared to the prior day's baseline.

\section{DISCUSSION}

This is the largest analysis of PB in infants to date, made possible through automated analysis of chest impedance waveforms throughout the NICU stay. The important findings are that preterm infants with higher GA spend more time in PB and that in some infants extreme $\mathrm{PB}$ or an abrupt increase over baseline was associated with illness, stress, or adverse events. This latter finding could have implications for predictive monitoring and early warning systems for hospitalized preterm infants.

\section{Distinguishing Features of Periodic Breathing Compared to AOP}

We found that $\mathrm{PB}$ is distinct from AOP in a number of ways, consistent with findings in other published studies $(2,29)$. Among 1,211 infants of $<35$ wk' gestation, nearly all had some PB. Infants born at 30-34 wk' gestation exhibited the highest proportion of time in $\mathrm{PB}$ (on average about 5\%), whereas infants with $<27$ wk' GA spent $<2 \%$ of the time in PB. PB tends to peak several weeks after birth and persists at NICU discharge. In contrast, $\mathrm{AOP}$ is more common and more severe in extremely low GA infants, begins shortly after birth, declines with advancing PMA, and typically resolves between 36 and
40 wk' PMA (see Figure 5 and companion paper on AOP). Others have reported that $\mathrm{PB}$ is more common in preterm compared to term infants, but these studies included very few extremely preterm infants $(29,30)$. Our data indicate that the proportion of time spent in $\mathrm{PB}$ follows an inverted U-shaped curve with respect to GA, with later preterm infants spending more time in this immature breathing pattern compared to extremely preterm or term infants.

The PB analyses we report are purely quantitative, and an important next step will be to perform both qualitative and quantitative analyses, including measuring the PB cycle duration, ratio of time in apnea relative to breathing, and respiratory rate and architecture of the breathing cycle (spindle, decrescendo, presence of a sigh breath at the beginning or end). These features may be important in determining whether PB is typical or atypical $(29,31,32)$, and if atypical whether it is linked to adverse events. Further studies are also needed to determine whether decreases in heart rate and oxygen saturation that are sometimes seen with the apneic phase of $\mathrm{PB}$ are associated with adverse neurologic outcomes or other morbidities.

\section{Mechanisms of PB in Neonates}

The mechanism of $\mathrm{PB}$ in preterm infants is purported to be heightened sensitivity or gain in chemoreceptors, resulting in overcompensation for small changes in $\mathrm{PaO}_{2}$ or $\mathrm{PaCO}_{2}$ and oscillation between brief periods of tachypnea and apnea $(11,25,33,34)$. At birth, peripheral chemoreceptors are silenced due to the sudden increase from fetal to neonatal blood oxygen levels (12). In the first weeks after birth, these receptors regain their sensitivity to hypoxia, coincident with the emergence and peak of PB. Central chemoreceptors have also been reported to play a role in $\mathrm{PB}$, with studies showing that the threshold for $\mathrm{CO}_{2}$ responsiveness is narrower in neonates compared to adults. In a study of preterm and term neonates, the difference between apneic and eupneic $\mathrm{CO}_{2}$ thresholds was about 1 Torr, in contrast to adults with a difference of about 3.5 Torr, which might partially account for neonates' tendency to oscillate between apnea and breathing $(33,35)$.

Our observation that extremely preterm infants have less $\mathrm{PB}$ than higher GA preterm infants may be due to use of continuous positive airway pressure and caffeine which limit PB $(36,37)$. Most extremely preterm infants are receiving these therapies at 2-4 weeks' chronologic age when chemoreceptors have the highest gain which is thought to increase $\mathrm{PB}$. We did not see an overall increase in PB in these infants at later PMAs when continuous positive airway pressure and caffeine are stopped, but we did find several infants with an acute increase in PB after caffeine discontinuation. Sleep state maturation may also play a role, since $\mathrm{PB}$ occurs predominately during quiet sleep which constitutes a smaller fraction of total sleep time at earlier PMAs.

\section{Associations With Extreme PB}

After establishing normative data we sought to determine clinical associations with extreme PB. A major impetus for this investigation was the death of two infants from our 


\section{Articles | Patel et al.}
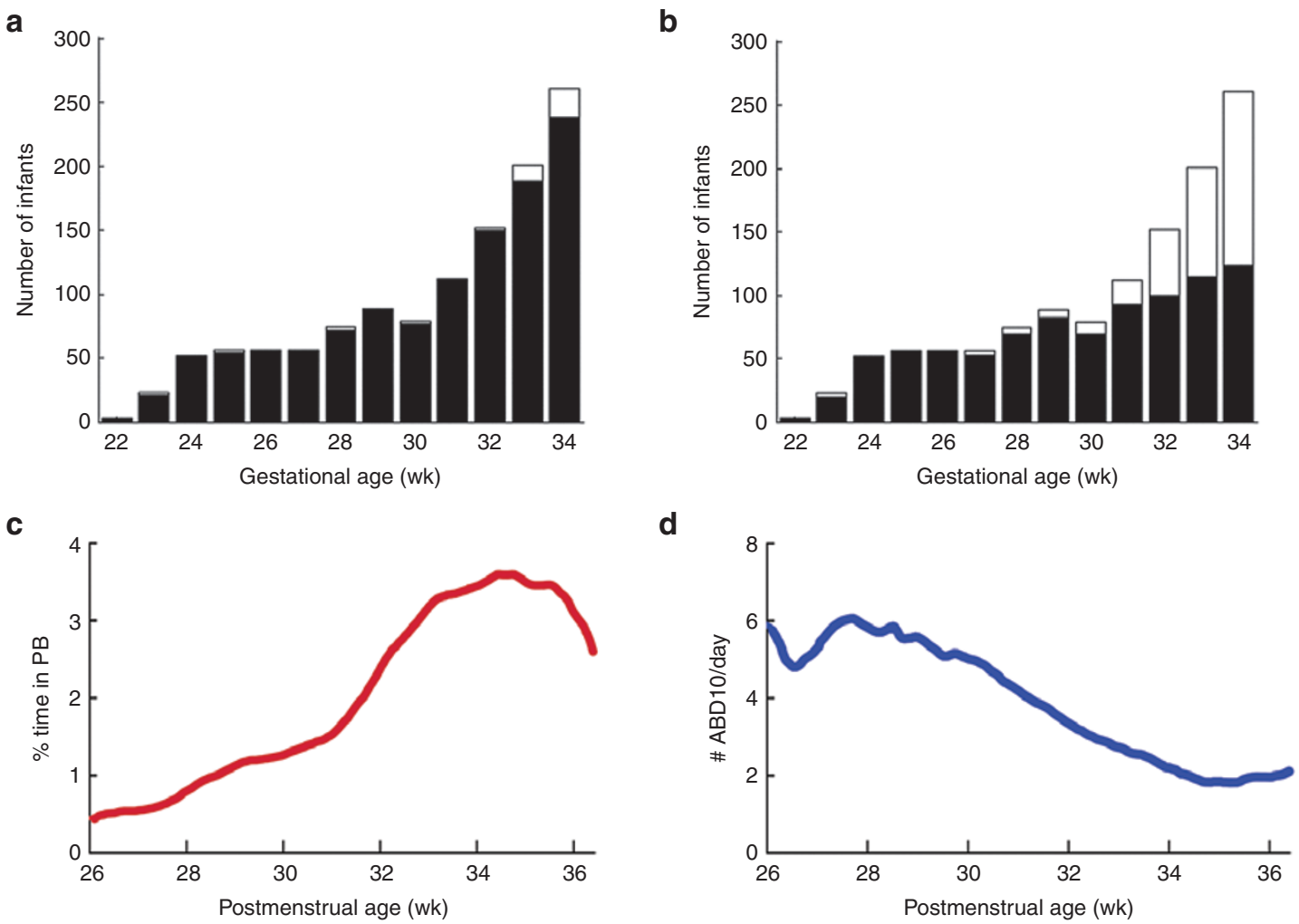

Figure 5 Incidence and timing of PB compared to apnea of prematurity. We analyzed PB and apnea of prematurity (AOP) in the same 1,211 preterm infants $<35$ wk' GA over the same time period in the NICU ( 53 infant-years' data). "ABD10's", apnea at least 10 s with associated bradycardia < 100 beats/ min and oxygen desaturation $<80 \%$, were analyzed using a validated automated algorithm (27), as described in the companion paper on AOP. (a) Number of infants in each GA week, with (black, $n=1,165)$ ) or without (white, $n=46$ ) PB detected. (b) Number of infants in each GA week with at least one ABD10 event detected (black, $n=887$ ) or with no ABD10s detected (white, 324). (c) Mean percent of each day spent in PB at 26-36 wk PMA. (d) Mean number of ABD10s per day of data from 26 to $36 \mathrm{wk}^{\prime} \mathrm{PMA}$.

NICU who, on retrospective review, spent a surprisingly large amount of time in PB. One was a 32-wk infant who died of SIDS shortly after NICU discharge. As we previously reported, this otherwise healthy infant was recognized, retrospectively, to have spent over fivefold more time in $\mathrm{PB}$ throughout the NICU stay compared to the average of all GA-matched infants, yet had an uncomplicated NICU course and no AOP documented in the medical record (26). Other studies have found that infants with apparent life-threatening events and siblings of infants that died of SIDS spend more time in PB compared to age-matched controls (38-40). SIDS is a rare and multifactorial event, and, practically speaking, we would estimate that analysis of chest impedance and vital sign data from about 300,000 infants $<35 \mathrm{wk}$ ' gestation through their NICU stay would be required to determine whether a particular respiratory or cardiorespiratory pattern is associated with SIDS.

We found extreme PB in some infants prior to diagnosis of septicemia and NEC. In one case, an infant experienced a $>10$-fold increase in PB over her prior baseline (from $<5$ to $>50 \%$ of the time in $\mathrm{PB}$ ) up to $18 \mathrm{~h}$ before she acutely decompensated and died of suspected sepsis. This was an exceptional case, but we did find a substantial number of infants with septicemia or NEC who experienced at least a twofold increase in \% $\mathrm{PB}$ in the day before diagnosis compared to their prior baseline. The increase in $\mathrm{PB}$ was more pronounced in cases of NEC than in LOS which may be due, in part, to the fact that NEC occurred in infants of higher GA and at later PMA, when PB tends to peak. Increased apneic spells have been described around the time of sepsis in preterm infants, likely in part due to cytokine-mediated release of prostaglandins which bind to receptors in respiratory centers of the brainstem and reduce respiratory drive (41-43). Further studies are warranted to determine whether realtime analysis and display of apnea and $\mathrm{PB}$ indices at the bedside is feasible, cost effective, and improves outcomes by identifying preterm NICU patients in the early stages of potentially catastrophic illnesses.

While some instances of extreme PB were associated with adverse events, others had no apparent clinical correlate or were temporally associated with administration of immunizations. Several studies have reported increased cardiorespiratory events in preterm infants after immunizations, but others have not found this association, which may reflect different maturational stages at the time of administration, different vaccine combinations, or variations in methods for assessing apnea (44-46). Nevertheless, it is quite possible that even a relatively minor stress such as immunizations may destabilize preterm infants causing them to revert to immature breathing patterns of apnea or PB. 


\section{Conclusion}

While $\mathrm{PB}$ is a normal immature breathing pattern for neonates, excessive $\mathrm{PB}$, or an abrupt increase over prior baseline warrants consideration for potential pathology. It remains to be determined whether quantitative and qualitative analysis of PB during the NICU stay can identify infants at high risk for adverse events who might benefit from preemptive therapies.

\section{METHODS}

\section{Study Population and Clinical Data Collection}

The University of Virginia Institutional Review Board approved the study which included all infants $<35 \mathrm{wk}$ gestation admitted to the UVa Neonatal Intensive Care Unit from January 2009 to March 2014. The study was purely observational and data were deidentified, therefore consent was waived. Decisions about clinical care including respiratory support were made by the medical team without knowledge of algorithm-detected apnea or PB. Generally, caffeine was given to all infants until they reached at least $32 \mathrm{wk}$ ' PMA and were having few or no events requiring intervention. Infants were generally transitioned from continuous positive airway pressure to high and then low flow nasal cannula oxygen, and $\mathrm{SpO}_{2}$ target range was $88-95 \%$ for infants on supplemental oxygen.

Medical records were reviewed for demographic variables, mortality and morbidities, and clinical events. Late-onset septicemia was defined as signs of sepsis $>3 \mathrm{~d}$ from birth, positive blood culture and a minimum of 5 days of antibiotic therapy. NEC was defined as clinical abdominal signs with radiographic presence of pneumatosis, portal venous air or pneumoperitoneum, or the need for surgery. Cases of NEC with associated septicemia were classified as NEC alone. Severe intraventricular hemorrhage was defined as grade III-IV, and bronchopulmonary dysplasia was defined as requirement for supplemental oxygen at 36 wk' PMA.

\section{Monitor Data Collection and Periodic Breathing Analysis}

Bedside monitor waveform and vital sign data, collected continuously on all infants in the NICU were stored for analysis using the BedMaster central network server (Excel Medical, Jupiter, FL). Waveform data included chest impedance collected at $60 \mathrm{~Hz}$ and three leads of electrocardiogram collected at $240 \mathrm{~Hz}$. Vital sign data included every $2 \mathrm{~s}(0.5 \mathrm{~Hz})$ heart rate, respiratory rate, and oxygen saturation $\left(\mathrm{SpO}_{2}\right) . \mathrm{SpO}_{2}$ averaging time was $8 \mathrm{~s}$.

We used our automated apnea detection system to analyze chest impedance signals and detect brief apneic pauses $(28,27)$. As previously reported, a wavelet transformation was performed to identify $\mathrm{PB}$ as $\geq 3$ respiratory pause/breath cycles of duration $10-40 \mathrm{~s}$. Clinicians independently reviewed chest impedance and vital signs, and a wavelet coefficient of 0.6 was determined to detect PB with $96 \%$ sensitivity and $90 \%$ specificity and to distinguish $\mathrm{PB}$ from irregular clusters of apnea (26).

Percent of time in $\mathrm{PB}(\% \mathrm{~PB})$ was calculated hourly for all times when chest impedance data were available and infants were not on mechanical ventilation. Mean \%PB for each week of GA and PMA were analyzed for the population to construct a heat map. We identified extreme periodic breathing as 12 -h periods in which there were at least $6 \mathrm{~h}$ of data available for analysis and the $\% \mathrm{~PB}$ was $>10 \%$ and more than 6 SDs above the mean for GA and PMA $(z$-score $>6)$. We also randomly selected a control group of the same number of infants without extreme PB, matched for GA and PMA. Medical records were reviewed around time periods with and without extreme $\mathrm{PB}$ for the following events: suspected or proven LOS or NEC within $24 \mathrm{~h}$, surgery or immunizations within $24 \mathrm{~h}$ prior, and caffeine discontinued within $8 \mathrm{~d}$ prior.

To compare $\mathrm{PB}$ to apnea of prematurity (AOP) in the same infants over the same time periods, we also analyzed central apnea events lasting at least $10 \mathrm{~s}$ and associated with bradycardia $<100$ beats/min and desaturation with $\mathrm{SpO} 2<80 \%$ (ABD10), using a previously validated algorithm (28). This analysis is described in a companion paper (Fairchild et al., (27)).

\section{Statistical Analysis}

Characteristics and outcomes of infants with and without extreme PB were compared using rank sum or Fischer exact tests for continuous or categorical variables, respectively. Amount of $\mathrm{PB}$ in the $2 \mathrm{~d}$ prior to septicemia or NEC diagnosis was compared using a signed rank test. Two-tailed significance was set at $P<0.05$, and analyses were performed in MATLAB (Mathworks, Natick, MA).

\section{STATEMENT OF FINANCIAL SUPPORT}

This study was supported by the National Institutes of Health grants HD072071 and HD064488.

Disclosure: None.

\section{REFERENCES}

1. Shannon DC, Carley DW, Kelly DH. Periodic breathing: quantitative analysis and clinical description. Pediatr Pulmonol 1988;4:98-102.

2. Barrington KJ, Finer NN. Periodic breathing and apnea in preterm infants. Pediatr Res 1990;27:118-21.

3. Chernick V, Heldrich F, Avery ME. Periodic breathing of premature infants. J Pediatr 1964;64:330-40.

4. Chernick V. 50 years ago in the Journal of Pediatrics: periodic breathing of premature infants. J Pediatr 2014;164:541.

5. Kelly DH, Riordan L, Smith MJ. Apnea and periodic breathing in healthy full-term infants, 12-18 months of age. Pediatr Pulmonol 1992;13:169-71.

6. Finer NN, Barrington KJ, Hayes B. Prolonged periodic breathing: significance in sleep studies. Pediatrics 1992;89:450-3.

7. Poets CF. Apnea of prematurity. In: Principles and Practice of Pediatric Sleep Medicine: Second Edition. Sheldon, Kryger, Ferber, Gozal (eds.). Philadelphia, PA: Elsevier., 2012:195-200.

8. Al-Matary A, Kutbi I, Qurashi M, et al. Increased peripheral chemoreceptor activity may be critical in destabilizing breathing in neonates. Semin Perinatol 2004;28:264-72.

9. Canet E, Praud JP, Bureau MA. Periodic breathing induced on demand in awake newborn lamb. J Appl Physiol (1985) 1997;82:607-12.

10. Darnall RA. The role of $\mathrm{CO}(2)$ and central chemoreception in the control of breathing in the fetus and the neonate. Respir Physiol Neurobiol 2010;173:201-12.

11. Edwards BA, Sands SA, Berger PJ. Postnatal maturation of breathing stability and loop gain: the role of carotid chemoreceptor development. Respir Physiol Neurobiol 2013;185:144-55.

12. Gauda EB, Carroll JL, Donnelly DF. Developmental maturation of chemosensitivity to hypoxia of peripheral arterial chemoreceptors-invited article. Adv Exp Med Biol 2009;648:243-55.

13. Katz-Salamon M. Delayed chemoreceptor responses in infants with apnoea. Arch Dis Child 2004;89:261-6.

14. Poets CF, Southall DP. Patterns of oxygenation during periodic breathing in preterm infants. Early Hum Dev 1991;26:1-12.

15. Razi NM, DeLauter M, Pandit PB. Periodic breathing and oxygen saturation in preterm infants at discharge. J Perinatol 2002;22:442-4.

16. Urlesberger B, Pichler G, Gradnitzer E, Reiterer F, Zobel G, Müller W. Changes in cerebral blood volume and cerebral oxygenation during periodic breathing in term infants. Neuropediatrics 2000;31:75-81.

17. Decima PF, Fyfe KL, Odoi A, Wong FY, Horne RS. The longitudinal effects of persistent periodic breathing on cerebral oxygenation in preterm infants. Sleep Med 2015;16:729-35.

18. Khoo MC, Anholm JD, Ko SW, et al. Dynamics of periodic breathing and arousal during sleep at extreme altitude. Respir Physiol 1996;103:33-43.

19. Tomita $Y$, Kasai $T$, Kisaka $T$, et al. Altered breathing syndrome in heart failure: newer insights and treatment options. Curr Heart Fail Rep 2015;12:158-65.

20. Rowat AM, Dennis MS, Wardlaw JM. Central periodic breathing observed on hospital admission is associated with an adverse prognosis in conscious acute stroke patients. Cerebrovasc Dis 2006;21:340-7.

21. Siccoli MM, Valko PO, Hermann DM, Bassetti CL. Central periodic breathing during sleep in 74 patients with acute ischemic stroke - neurogenic and cardiogenic factors. J Neurol 2008;255:1687-92.

22. Corrà U, Pistono M, Mezzani $\mathrm{A}$, et al. Sleep and exertional periodic breathing in chronic heart failure: prognostic importance and interdependence. Circulation 2006;113:44-50. 


\section{Articles | Patelet al.}

23. Cherniack NS, Longobardo GS. Mathematical models of periodic breathing and their usefulness in understanding cardiovascular and respiratory disorders. Exp Physiol 2006;91:295-305.

24. Millar TW, Hanly PJ, Hunt B, Frais M, Kryger MH. The entrainment of low frequency breathing periodicity. Chest 1990;98:1143-8.

25. Khoo MC, Kronauer RE, Strohl KP, Slutsky AS. Factors inducing periodic breathing in humans: a general model. J Appl Physiol Respir Environ Exerc Physiol 1982;53:644-59.

26. Mohr MA, Fairchild KD, Patel M, et al. Quantification of periodic breathing in premature infants. Physiol Meas 2015;36:1415-27.

27. Fairchild K, Mohr M, Paget-Brown A, et al. Clinical associations of immature breathing in preterm infants: part 1—central apnea. Pediatr Res e-pub ahead of print 9 March 2016.

28. Lee H, Rusin CG, Lake DE, et al. A new algorithm for detecting central apnea in neonates. Physiol Meas 2012;33:1-17.

29. Glotzbach SF, Baldwin RB, Lederer NE, Tansey PA, Ariagno RL. Periodic breathing in preterm infants: incidence and characteristics. Pediatrics 1989;84:785-92.

30. Oliveira AJ, Nunes ML, Fojo-Olmos A, Reis FM, da Costa JC. Clinical correlates of periodic breathing in neonatal polysomnography. Clin Neurophysiol 2004;115:2247-51.

31. Wilkinson MH, Skuza EM, Rennie GC, et al. Postnatal development of periodic breathing cycle duration in term and preterm infants. Pediatr Res 2007;62:331-6.

32. Barrington KJ, Finer NN, Wilkinson MH. Progressive shortening of the periodic breathing cycle duration in normal infants. Pediatr Res 1987;21:247-51.

33. Khan A, Qurashi M, Kwiatkowski K, Cates D, Rigatto H. Measurement of the $\mathrm{CO} 2$ apneic threshold in newborn infants: possible relevance for periodic breathing and apnea. J Appl Physiol (1985) 2005;98:1171-6.

34. Darnall RA, Ariagno RL, Kinney HC. The late preterm infant and the control of breathing, sleep, and brainstem development: a review. Clin Perinatol 2006;33:883-914; abstract $\mathrm{x}$.
35. Pereira MR, Reis FC, Landriault L, Cates DB, Rigatto H. Profile of alveolar gases during periodic and regular breathing in preterm infants. Biol Neonate 1995;67:322-9.

36. Edwards BA, Sands SA, Feeney C, et al. Continuous positive airway pressure reduces loop gain and resolves periodic central apneas in the lamb. Respir Physiol Neurobiol 2009;168:239-49.

37. Glotzbach SF, Tansey PA, Baldwin RB, Ariagno RL. Periodic breathing in preterm infants: influence of bronchopulmonary dysplasia and theophylline. Pediatr Pulmonol 1989;7:78-81.

38. Kelly DH, Shannon DC. Periodic breathing in infants with near-miss sudden infant death syndrome. Pediatrics 1979;63:355-60.

39. Kelly DH, Walker AM, Cahen L, Shannon DC. Periodic breathing in siblings of sudden infant death syndrome victims. Pediatrics 1980;66:515-20.

40. Hunt CE, Corwin MJ, Lister G, et al.; Collaborative Home Infant Monitoring Evaluation (CHIME) Study Group. Precursors of cardiorespiratory events in infants detected by home memory monitor. Pediatr Pulmonol 2008;43:87-98.

41. Hofstetter AO, Legnevall L, Herlenius E, Katz-Salamon M. Cardiorespiratory development in extremely preterm infants: vulnerability to infection and persistence of events beyond term-equivalent age. Acta Paediatr 2008;97:285-92.

42. Hofstetter AO, Saha S, Siljehav V, Jakobsson PJ, Herlenius E. The induced prostaglandin E2 pathway is a key regulator of the respiratory response to infection and hypoxia in neonates. Proc Natl Acad Sci USA 2007;104:9894-9.

43. Balan KV, Kc P, Hoxha Z, Mayer CA, Wilson CG, Martin RJ. Vagal afferents modulate cytokine-mediated respiratory control at the neonatal medulla oblongata. Respir Physiol Neurobiol 2011;178:458-64.

44. Furck AK, Richter JW, Kattner E. Very low birth weight infants have only few adverse events after timely immunization. J Perinatol 2010;30:118-21.

45. Carbone T, McEntire B, Kissin D, et al. Absence of an increase in cardiorespiratory events after diphtheria-tetanus-acellular pertussis immunization in preterm infants: a randomized, multicenter study. Pediatrics 2008;121:e1085-90.

46. DeMeo SD, Raman SR, Hornik CP, Wilson CC, Clark R, Smith PB. Adverse events after routine immunization of extremely low-birth-weight infants. JAMA Pediatr 2015;169:740-5. 